\title{
Enhanced of the R6G Thermal Diffusivity on Aggregated Small Gold Particles
}

\author{
J. L. Jiménez Pérez ${ }^{(a)}$, J. F. Sánchez Ramírez ${ }^{(a)}$, R. Gutiérrez Fuentes $^{(a)}$, A. Cruz-Orea $^{(b)}$, and J. L. Herrera Pérez ${ }^{(a)}$ \\ (a) CICATA-IPN, Legaria 694, Col. Irrigación, 11500 México D.F. México \\ (b) Depto. de Física, CINVESTAV-IPN, A.P. 14-740, 07360 México D.F. México
}

Received on 4 December, 2005

\begin{abstract}
R6G dye with gold nanoparticles was prepared in water solution. Using a thermal lens experimental setup, the thermal diffusivity of R6G in presence of gold nanoparticles with Au different concentration was measured. The results show that, the thermal diffusivity of R6G dye mixed with gold nanoparticles increases with the increase of particle metallic concentration, enhancing the thermal diffusivity in the solution. The thermal diffusivity of the samples was studied for a constant concentration of R6G dye $0.1 \mathrm{~g} / \mathrm{L}$. The diffusivities were obtained by using the thermal lens aberrant model with lasers arranged in the mismatched mode. The characteristic time constant of the transient thermal lens was obtained by fitting the theoretical expression, for transient thermal lens, to the experimental data. UV-Vis spectroscopy and TEM were used to characterize the R6G dye gold nanoparticles.
\end{abstract}

Keywords: Thermal diffusivity; Nanofluids; Gold nanoparticles; Thermal lens

\section{INTRODUCTION}

In the last three decades, we have witnessed the development of a technique for nondestructive characterization of the thermal, optical and structural properties of materials based upon the photothermal techniques such as the photothermal lensing spectroscopy. In this method, the signal magnitude is directly related to temperature rise in a light-illuminated material and to the amount of heat generated via optical absorption and subsequent nonradiative relaxation. This thermal lensing TL is a versatile and viable technique for measuring thermal diffusivities of semitransparent materials taking place in organic materials and dyes (that are widely used in biology and medicine for staining biological objects for diagnostic and therapeutic purposes). The discovery of photothermal lensing effect allowed the monitoring of nonradiative relaxation in excited molecule and it has been refined by various researchers to suite for study of various phenomena related to light-matter interactions $[1,2]$. Most important modification of the thermal lens technique is the aberrant method developed by Shen et. al. [3]. With this method one can record the thermal lens spectrum of semitransparent samples. One of the important features of this technique is that it is a highly sensitive method for measuring the thermal diffusivity in a multitude of samples based on physical changes that occur in the sample.

Traditional heat transfer fluids, including oil, water and dyes mixture are poor heat transfer fluids. Because the thermal diffusivity of these fluids plays an important role in development of energy-efficient heat transfer equipment, numerous methods have been taken to improve the thermal diffusivity of these fluids. Therefore, fluids containing suspended solid nanoparticles can reasonably be expected to display significantly enhanced thermal diffusivity relative to those of pure liquids. However, nanoparticles appear to be ideally suited for applications in targeted thermal effects in medical therapies and photo thermally activated drug delivery, all depend critically on the thermal transport between the nanoparticles and the surrounding liquid. Nanofluid containing nanoparticles exhibit enhanced thermal conductivity. For example of such versatility of the heat transfer fluids, we can mention the ethylene glycol (EG) nanofluid containing $\mathrm{CuO}$ nanoparticles exhibit enhanced thermal diffusivity. For example, a maximum increase in the thermal diffusivity of approximately 22 $\%$ was observed in a $\mathrm{CuO} / \mathrm{EG}$ nanofluid contained $4 \%$ in vol. of $\mathrm{CuO}$ nanoparticles with the average diameter of $23.6 \mathrm{~nm}$ [4].

In this paper we used a thermal lens experimental setup to obtain the thermal diffusivity of R6G in presence of gold nanoparticles with Au different concentrations. The diffusivities will be obtained by using the thermal lens aberrant model with lasers arranged in the mismatched mode. The characteristic time constant of the transient thermal lens was obtained by fitting the experimental data to the theoretical expression for transient thermal lens. UV-Vis spectroscopy and TEM were used as complementary techniques in the characterization of the R6G dye gold nanoparticles.

\section{THEORY}

When a medium is illuminated with Gaussian laser radiation, some of the incident energy is absorbed by molecules in the ground state, and are excited to higher energy states. The excess energy acquired by the molecules can be dissipated in many ways. The non-radiative decay produces localized temperature rise, creating a refractive index gradient in the medium so that the medium acts as a lens-like optical element, called thermal lens. The theoretical treatment of the thermal lens effect takes into account the spherical aberration of the thermal lens and also considers the whole optical path length change with temperature. The Shen's model [5] considers that a heat source is induced by the laser beam, that is proportional to the Gaussian intensity profile, and can be expressed as: $\mathrm{I}_{e}(\mathrm{r})=\left(2 \mathrm{P}_{e} / \pi \omega_{e}^{2}\right) \exp \left(-2 \mathrm{r}^{2} \omega_{e}^{2}\right)$, where $\mathrm{P}_{e}$ is the excitation beam power and $\omega_{e}$ is the excitation beam waist at the sample position . The solution of the heat conduction equation depends on the employed boundary conditions. This theory developed the infinitive aberrant model for the mode mis- 
matched configuration. The temporal evolution of the temperature profile $T(r, t)$ induced by the TL, in the sample is given by [3]:

$\Delta T(r, t)=\frac{2 P_{e} A_{e}}{\pi c \rho \omega_{e}^{2}} \int_{0}^{t} I_{0}\left(\frac{1}{1+\left(2 t^{\prime} t_{c}\right)}\right) \exp \left(\frac{2 r^{2}}{\omega_{e}^{2}} 1+\left(2 t^{\prime} t_{c}\right)\right) d t^{\prime}$

where $\rho$ is the density, $c$ the specific heat, $A_{e}$ is the optical absorption coefficient, at the excitation beam wavelength, and $t_{c}$ is the characteristic TL time constant, defined as:

$$
t_{c}=\frac{\omega_{e}^{2}}{4 D}
$$

where $D$ is the thermal diffusivity. As it was mentioned previously this temperature rise, which carries a Gaussian profile, induces a slight distortion in the probe beam wave front that can be associated with the change in the sample refractive index with respect to beam axis, as follows:

$$
\phi=\frac{2 \pi}{\lambda_{p}} \ell_{0}\left(\frac{d n}{d T}\right)_{p}[\Delta T(r, t)-\Delta T(0, t)]
$$

where $\varnothing$ is the phase shift induced when the prove beam passes through the TL, $\lambda_{p}$ is the probe beam wavelength, $l_{0}$ is the sample thickness and $(d n / d T)_{p}$ is the temperature dependence of the sample refractive index. Finally, using the Fresnell diffraction theory, the probe beam intensity at the detector plane can be written as an analytical expression for the absolute determination of the thermo-optical properties to the sample, as:

$I(t)=I(0)\left[1-\frac{\theta}{2} \tan ^{-1}\left(\frac{2 m V}{\left[(1+2 m)^{2}+V^{2}\right] \frac{t_{c}}{2 t}+1+2 m+V^{2}}\right)\right]^{2}$

where

$$
m=\left(\frac{\omega_{1 p}}{\omega_{e}}\right)^{2} ; \quad V=\frac{Z_{1}}{Z_{c}} ; \quad \theta=-\frac{P_{e} A_{e} l_{0}}{k \lambda_{p}}\left(\frac{d n}{d T}\right)_{p}
$$

In Eq. (4) $I(t)$ is the temporal dependence of the probe laser beam intensity at the detector, $I(0)$ is the initial value of $I(t)$, $\theta$ is the thermally induced phase shift of the beam after its passing through the sample, $Z_{c}$ is the confocal distance of the probe beam, and $Z_{1}$ is the distance of the probe beam waist from to sample.

\section{EXPERIMENTAL}

Fluids containing Gold $(\mathrm{Au})$ nanoparticles were prepared according to the method described by Park et al. [5], with some modifications. In a typical synthesis, solutions of $\mathrm{HAuCl}_{4}(0.033 \mathrm{mmol}$ in $25 \mathrm{ml}$ of water) and poly (N-vinyl-2pyrrolidone) (PVP, $30 \mathrm{mg}$ in $20 \mathrm{ml}$ of water) were prepared by dissolving the $\mathrm{HAuCl}_{4}$ crystals and PVP in water. Both solutions were mixed to produce an $\mathrm{Au}$ (III) ion solution containing PVP as a protective polymer. Then an aqueous solution of ascorbic acid (AA, $0.066 \mathrm{mmol}$ in $5 \mathrm{ml}$ of water) was added to the resulting solution at room temperature. A fluid containing metallic particles was formed after the addition of AA in the mixture solution. A known concentration of Rh-6G in water was mixed with the fluids containing Au nanoparticles to produce dye-Au nanoclusters assemblies with different R6G/Au (156.0, 75.7, 18.9, 9.46, 6.31, 4.73 and 3.78) molar ratio in 10 $\mathrm{ml}$ of water. The fluids then were placed in a quartz cuvette with $1 \mathrm{~cm}$ thick for the optical and thermal measurements. The experiments were performed at room temperature.

A Shimadzu UV-VIS 3101PC double beam spectrophotometer was used to record the absorption spectra of fluids. Particle sizes and size distribution were evaluated by TEM, using a JEOL-JEM200 microscope. For TEM observations, a drop of fluids was spread on a carbon-coated copper microgrid and dried subsequently in vacuum. Gold particles with average size ranging from $13.9 \mathrm{~nm}$ to $37.4 \mathrm{~nm}$ were measured The TL effect of such nanofluids was based on their laser-induced heating and time resolved monitoring on the thermal effects in nanofluids.

\section{RESULTS AND DISCUSSION}

A typical TEM micrograph of fluids containing Au nanoparticles and its optical absorption spectrum are shown in Fig. 1. The distribution of the Au nanoparticles is also presented for the first R6G/Au ratio. The absorption spectrum of the fluids revealed a surface plasmon resonance (SPR) band with a peak around $529 \mathrm{~nm}$ (figure $1 \mathrm{~b}$ ). Formation of nanoparticles is clear from the TEM micrographs. The average size of the particles was $37.4 \mathrm{~nm}$. Similar electron micrographs and particle size histograms of Au nanoparticles (not shown here) prepared with the different ratios of metal ion concentrations R6G/Au were obtained in $50 \mathrm{ml}$ of water.

The TL effect of such nanofluids was based on their laserinduced heating and time resolved monitoring on the thermal effects. The schematic diagram of the TL experimental setup is shown in references [6]. In this experimental set-up it was used an optical filter in front of the photo detector in order to avoid the fluorescent irradiation, from radiative relaxation, that comes from the R6G dye. In this way it was only detected the radiation of the prove laser beam of $\mathrm{He}-\mathrm{Ne}$, that takes into account only the non-radiative relaxation.

The time evolution of TL signal for nanoliquids samples, at room temperature, with $\mathrm{R} 6 \mathrm{G} / \mathrm{Au}$ ratios equal to 156.0 and 75.7 are shown in Figs. 2 and 3 respectively. The symbols (o) represent the experimental points and the solid lines correspond to the best fit of Eq. 4, to the experimental data, leaving $\theta$ and $t_{c}$ as adjustable parameters. Using $\mathrm{t}_{c}=\left(\omega_{e}^{2} / 4 \mathrm{D}\right)$ and having $\omega_{e}=4.0 \times 10^{-3} \mathrm{~cm}$, we obtained $\mathrm{D}=(15.6250 \pm 0.12207)$ $\times 10^{-4} \mathrm{~cm}^{2} / \mathrm{s}$, for the thermal diffusivity corresponding to the sample with R6G/Au ratio equal to 156.0. Fig. 3 shows the transient thermal lens signal for nanofluid $\mathrm{R} 6 \mathrm{G} / \mathrm{Au}$ ratio equal to 75.7. From the best fitting of Eq. 4 to the experimental 

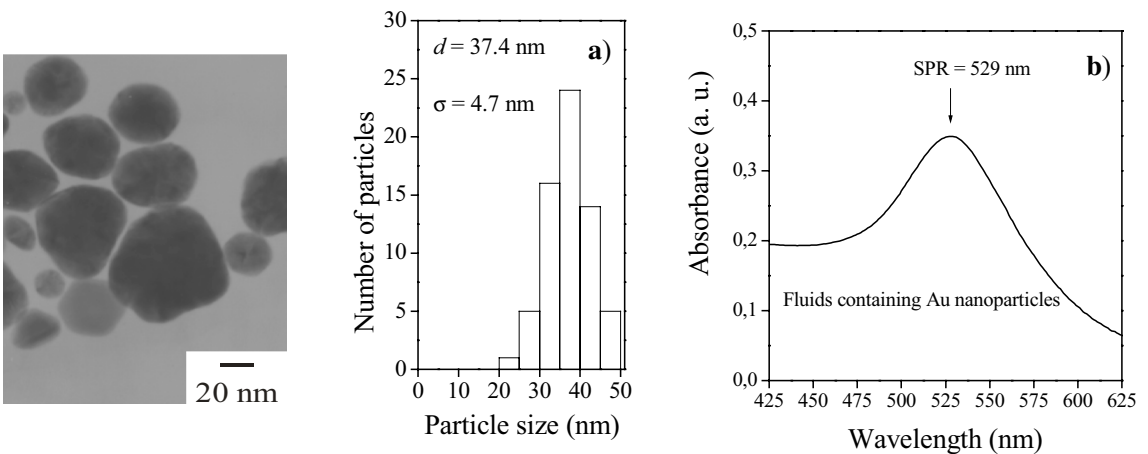

FIG. 1: a) A typical TEM micrograph of fluids containing Au nanoparticles with its size distribution. b) Optical absorption spectrum of fluids containing Au nanoparticles.

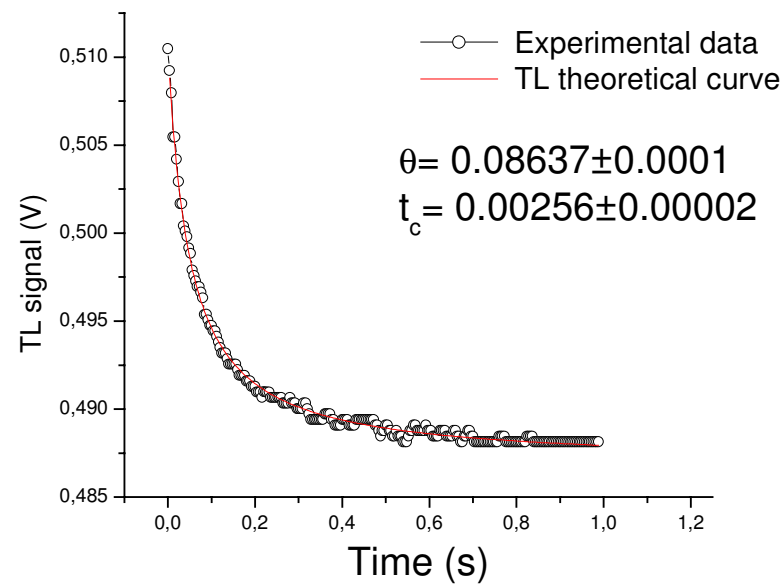

FIG. 2: Time evolution of the TL signal for gold nanoparticles (R6G/Au ratio equal to 156.0). Symbols represent the experimental data and the dotted line, beneath the symbols, the best fit with Eq. 4.

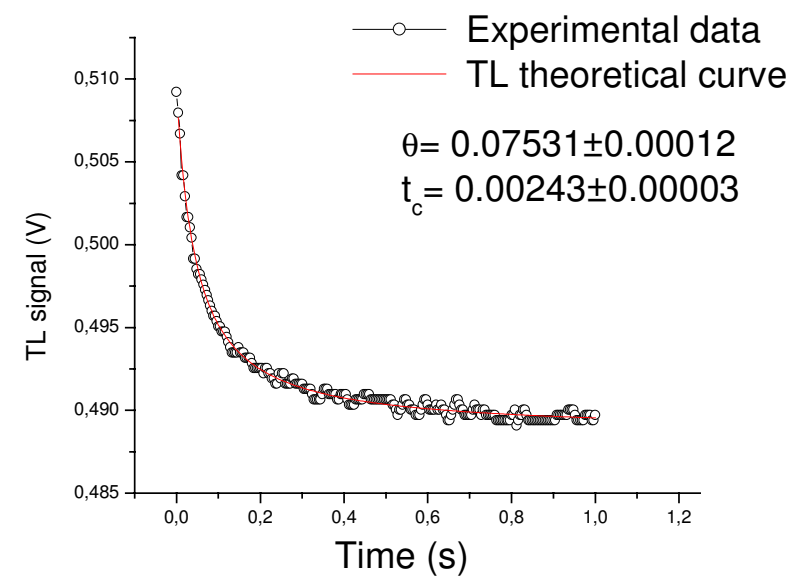

FIG. 3: Time evolution of the TL signal for gold nanoparticles (R6G/Au ratio equal to 75.7). Symbols represent the experimental data and the dotted line, beneath the symbols, the best fit with Eq. 4 . data, it was obtained the thermal diffusivity for the nanoliquid sample: $\mathrm{D}=(16.460905 \pm 0.203221) \times 10^{-4} \mathrm{~cm}^{2} / \mathrm{s}$. It is possible to see from Figs. 2 and 3 that the signal decreases with time, indicating that thermal lens is divergent, thus defocusing the probe beam on the detector. This behavior is due to the fact that the temperature coefficient of the optical path length, ds/dT, is negative for most of transparent liquids and plastics. Table I summarizes the thermal diffusivity values, obtained from the fit of Eq. 4 to the experimental data, for the solution of R6G in water and the other seven R6G/Au mentioned ratios. For the case of R6G in water it was obtained a thermal diffusivity value near to the value corresponding of water thermal diffusivity. Also from the Table 1 it can be seen that there is an increase in the fluid thermal diffusivity when the $\mathrm{R} 6 \mathrm{G} / \mathrm{Au}$ ratio decrease, that means the $\mathrm{Au}$ particle concentration increases. A possible explanation, of this increment in thermal diffusivity with the R6G/Au decrease, is given by Chandrasekharan et al. [7]. In this reference they mention that the R6G/Au assembly has absorption in $529 \mathrm{~nm}$ corresponding to the surface plasmon resonant (see Fig. 1 b), laser excitation would lead to the generation of hot electrons. These hot electrons are rapidly thermalized by electronphonon scattering. The energy deposit into the phonon modes is subsequently transferred to the surrounding medium which would increase the nanofluid thermal diffusivity.

\section{CONCLUSION}

Thermal diffusivity of rhodamine R6G dye with gold nanoparticles at different $\mathrm{R} 6 \mathrm{G} / \mathrm{Au}$ ratios, in water solution, was measured. The results show that the thermal diffusivity of R6G dye, mixed with gold nanoparticles, increased with the increase of $\mathrm{Au}$ particle concentration, enhancing the thermal diffusivity in the solution.

\section{Acknowledgment}

Financial support received from the Mexican agency Conacyt, CGPI and COFAA, México is gratefully acknowledged. A. Cruz-Orea is grateful for the financial support of CONACYT project No. 43252. 
TABLE I: Adjustable parameter $t_{c}$, obtained from the fit of Eq. (4) to the experimental data, and their calculated thermal diffusivity values for the solution of R6G in water and the different $\mathrm{R} 6 \mathrm{G} / \mathrm{Au}$ ratios.

\begin{tabular}{|l|l|l|}
\hline & $t_{C}(s)$ & $\begin{array}{l}\text { Thermal diffusivity } \\
\left(10^{-4} \mathrm{~cm}^{2} / \mathrm{s}\right)\end{array}$ \\
\hline R6G & $0.00306 \pm 0.00005$ & $13.071895 \pm 0.228828$ \\
\hline R6G/Au & & \\
\hline 156.0 & $0.00256 \pm 0.00002$ & $15.6250 \pm 0.12207$ \\
\hline 75.75 & $0.00243 \pm 0.00003$ & $16.460905 \pm 0.203221$ \\
\hline 18.92 & $0.00218 \pm 0.00003$ & $18.3486238 \pm 0.2525039$ \\
\hline 9.46 & $0.00209 \pm 0.00003$ & $19.1387559 \pm 0.2747189$ \\
\hline 6.31 & $0.00189 \pm 0.00003$ & $21.16402116 \pm 0.335936$ \\
\hline 4.73 & $0.00182 \pm 0.00002$ & $21.97802198 \pm 0.241516$ \\
\hline 3.78 & $0.00169 \pm 0.00001$ & $23.668639 \pm 0.1400511$ \\
\hline & & \\
\hline
\end{tabular}

[1] J. P. Gordon, R. C. C. Leite, R. S. Moore, S. P. S. Porto, and J. R. Whinnery, 36, 3 (1964).

[2] O. M. Wilson, K. Hu, D. G. Cahill, and P. V. Braun, Physics Review B 66, 224301 (2002).

[3] J. Shen, R. D. Lowe, and R. D. Snook, Chemical Physics 165: 385 (1992).

[4] Q. Xue, W. M. Xu, Mat. Chem. Phys., 90: 298 (2005).

[5] J. Park, V. Privman, E. Matijevic, J. Phys. Chem. B 105: 11630
(2001)

[6] R. Carbajal Valdez, A. Lomelí Mejia, J. L. Jiménez Pérez, A. Cruz Orea, and S. Tomás Velázquez, Rev. Sup. y Vac. 17, 7 (2004)

[7] N. Chandrasekharan, P. V. Kamat, J. Hu, and G. Jones II, J. Phys. Chem. B, 104, 11103 (2000). 